\title{
The impact of obesity on prostate cancer
}

\author{
Joep G. H. van Roermund · J. Alfred Witjes
}

Received: 27 February 2007 / Accepted: 30 April 2007 / Published online: 30 May 2007

(c) Springer-Verlag 2007

\begin{abstract}
Increasing prevalence of obesity in many parts of the world emphasizes the importance of learning more about the relationship between obesity and prostate cancer (PC). The present paper reviews the impact of obesity on $\mathrm{PC}$ using knowledge obtained from the available literature. Search of published literature in PUBMED database. Adipose tissue constitutes an active endocrine and metabolic organ which may be relevant in the development and progression of PC by different potential mechanisms. Furthermore, obesity could have an impact on the outcome of different treatment modalities for PC, both functionally as anatomically. Obesity is a growing problem, however, the exact role in the development and progression of PC has not been elucidated. Regarding the optimal treatment of PC in obese patients, comparative prospective studies are needed.
\end{abstract}

Keywords Obesity · Prostate cancer .

Body mass index (BMI)

\section{Introduction}

Obesity is one of the most challenging and growing health problems in industrialized countries. In Europe, the prevalence of obesity has more than doubled during the last two decades. According to the World Health Organization (WHO), obesity is defined as body mass index (BMI, weight in kilograms divided by height in meters squared,

J. G. H. van Roermund · J. A. Witjes ( $ه)$

Department of Urology, Radboud University Nijmegen

Medical Centre, PO Box 9101,

6500 HB Nijmegen, The Netherlands

e-mail: F.Witjes@uro.umcn.nl $\mathrm{kg} / \mathrm{m}^{2}$ ) over $30 \mathrm{~kg} / \mathrm{m}^{2}$. At present, more than half of all adults are categorized as having overweight (BMI, between 25 and $30 \mathrm{~kg} / \mathrm{m}^{2}$ ), and up to $30 \%$ is obese (BMI $>30 \mathrm{~kg} /$ $\mathrm{m}^{2}$ ) [1]. However, currently, there is no universally accepted measurement of obesity. BMI and waist-to-hip ratio (WHR) are the most common anthropometric methods used in clinical practice. Since abdominal visceral adipocytes are more metabolically active than abdominal subcutaneous adipocytes, one of the limitations of BMI is that it could be a less valid indicator of adiposity among the elderly, who tend to have a shift of fat from peripheral to central sites with an increase in WHR at a constant level of BMI [2]. More precisely WHR measures abdominal adipose tissue (or circumference) and fat distribution. The waist is defined as the abdominal circumference midway between costal margin and the crest of the iliac. The largest circumference just below the iliac crest is defined as the hip. For men a WHR $>0.90$ is a fairly accurate determination of an increased risk for obesity-related events.

Obesity is associated with numerous conditions that includes hypertension, high glucose levels, dyslipidemia, and central adiposity. This cluster of conditions refers to the metabolic syndrome [3]. This metabolic syndrome is accompanied by numerous chronic diseases, including hypertension, coronary artery disease, asthma, diabetes and arthritis, having a great impact on general health systems [4]. In addition to its link to several chronic medical conditions, obesity has also been associated with increased death rates of several types of cancer [5]. High prevalence of prostate cancer (PC) and increasing prevalence of obesity in many parts of the world, emphasizes the importance of learning more about the relationship between obesity and PC.

This review will look at the relationship between obesity and PC. Firstly, we briefly describe some of the hormonal 
sequelae of obesity and the different potential biological mechanisms affecting the prostate. Secondly, we review and discuss potential links between obesity and the development and detection of PC. Finally, the impact of obesity are summarized on surgical outcomes, and biochemical recurrence, following prostate surgery.

\section{Potential biochemical mechanisms affecting the developing of prostate cancer}

Adipose tissue actually is an active endocrine and metabolic organ, which could influence PC on several ways.

First, PC is a sex steroid sensitive disease. Obesity has an important impact on the synthesis and bioavailability of endogenous sex steroids and is associated with increased serum estradiol and decreased serum concentrations of free testosterone. Increased levels of estradiol are caused by peripheral conversion of testosterone to estradiol by increased aromatase activity secondary to the accumulation of adipose tissue. Obesity also reduces the concentrations of sex-hormone-binding globulin (SHBG). Both phenomena increase the fraction of bioavailable estradiol and lead to a reduction in total testicular testosterone production $[2$, 6]. At first sight this might seem protective for PC. However, reports have shown that a lower preoperative total testosterone correlated with a poorer pathological stage at time of surgery [7, 8]. Also, a large pooled case control prospective study found a modest, but significant decrease in PC risk for increasing levels of total testosterone [9].

In an experimental study, prenatal exposure to an extreme low dose of diethylstilbestrol (DES) and other estrogenic compounds significantly affects the development of mouse prostate in the presence of androgen. Another preliminary report suggests that offspring of DES-exposed mothers have a higher risk of prostate cancer [10]. Together, although estrogen is used as an anti-androgen in the treatment of advanced PC, these data suggest that estrogens may enhance the risk of PC earlier in life.

Second, beyond alterations in sex steroid hormones, obesity is also associated with increased levels of several other serum hormones, such as leptin, insulin and insulinlike growth factor-1 (IGF-1). Leptin, an obesity related hormone, is produced by adipocytes and plays a major role in controlling body weight homeostasis and is directly related to the degree of obesity. However, leptin receptors also have been identified in the prostate gland, suggesting a plausible biological role in this gland [11]. Recently, leptin has been shown to stimulate the in-vitro growth of hormone refractory PC cell lines $[12,13]$. In both reports leptin caused significant proliferation in both the PC-3 and DU145 cell lines when compared with untreated control cells. Clinical studies looking at the relation between leptin and PC are not consistent. A Chinese case-control study reported an increased risk of PC, but the trends were not statistically significant [14]. After adjustment for age, the men in the highest tertile of leptin levels had an approximately twofold risk of PC (Odds ratio, $\mathrm{OR}=1.78,95 \%$ $\mathrm{CI}=1.07-2.95)$. Albeit a Norwegian case-control study found no support for this hypothesis $(\mathrm{OR}=0.9,95 \%$ $\mathrm{CI}=0.6-1.6)$ [15]. Although the risk for PC in individuals with higher levels of leptin is not clearly proven, some studies showed that higher levels of leptin were linked to tumour progression and advanced disease. Saglam et al. [16] noticed in a cross-sectional study that elevated leptin was significantly associated with poorly differentiated cancer and extraprostatic cancer. Chang et al. [17] reported men with elevated plasma leptin concentrations had an increased risk of being diagnosed with high-volume PC that was attenuated after adjustment for BMI $(\mathrm{OR}=2.41,95 \%$ $\mathrm{CI}=0.93-4.58)$. One study did not find an relation between serum leptin and pathological stage (pT3a). However this study was done in predominantly white men with mainly low risk disease $(\mathrm{OR}=1.14,95 \% \mathrm{CI}=0.76-1.71)$ [18]. In all, these observations suggest that leptin may play no role in initiation of PC but may play some part in the progression of PC.

Diabetes mellitus (non-insulin dependent, type-II) and obesity are common conditions which are components of the same metabolic syndrome. In obesity, different endocrine and metabolic signals lead to insulin resistance, resulting in a chronic compensatory hyperinsulinaemia and increased levels of bioavailable IGF-1. In their turn, insulin and IGF-1 promote cellular proliferation and inhibit apoptosis in many tissue types [5]. These effects may be responsible for tumorgenesis of PC in obese patients. In an invitro study a significantly lower proliferation rate of androgen-independent PC-3 prostate cells was seen in IGF-1 deficient hosts in comparison to IGF-1 expressing hosts [19]. In addition, several prospective cohort and case-control studies have shown positive associations between PC risk and circulating IGF-1 level in men [20-22]. In these studies the OR varied between $1.63(95 \% \mathrm{CI}=0.92-2.89)$ and $4.3(95 \% \mathrm{CI}=1.8-10.6)$ for men in the highest quartile of IGF-I.

In summary, possible mechanisms by which obesity may impact upon PC include altered serum steroid hormones, leptin, insulin and IGF-1, levels. Most studies of hormone levels and PC risk have evaluated each hormone individually. Difficulties comparing these studies are caused by complex interrelationships between hormones, binding proteins and their receptors and the moment of exposure. In addition, most studies on obesity usually reflect an onemoment evaluation, whereas the cumulative effect of fat content (all-life exposure) may be relevant in prostatic disease development. 


\section{Risk of developing prostate cancer in obese patients}

Two large retrospective cohort studies found a positive relation between BMI and PC. One among 135,000 Swedish construction workers, reported a positive association of BMI $\left(>26.2 \mathrm{~kg} / \mathrm{m}^{2}\right)$ and risk of PC $(\mathrm{RR}=1.13,95 \%$ $\mathrm{CI}=0.99-1.29, P=0.10)$ [23]. The association between $\mathrm{BMI}$ and $\mathrm{PC}$ was stronger when looking at mortality than at incidence $(\mathrm{RR}=1.40,95 \% \mathrm{CI}=1.09-1.81, P=0.04)$. The other, a large Norwegian cohort study of 950,000 men, reported an increasing risk of PC with increasing BMI in men having a BMI below $35.0 \mathrm{~kg} / \mathrm{m}^{2}(\mathrm{RR}=1.15,95 \%$ $\mathrm{CI}=1.03-1.28)$ [24]. Interestingly, higher obesity-associated risk was seen in those being youngest with an age between 20 and 29 years at measurement. On the other hand, two more recent case-control studies addressed a protective association between obesity and PC risk [25, 26]. The age adjusted odds ratios ranged from 0.78 (95\% $\mathrm{CI}=0.56-1.09)$ to 0.91 (95\% $\mathrm{CI}=0.66-1.21)$. Finally, another recent prospective population-based cohort study even found no association [27]. It should be noted that nonbiological explanations may contribute to the apparent disparate results among epidemiological studies. For example, some studies were done before the PSA era, different BMI categorizations were used, and study populations differed in sample size.

Another problem is that most of these studies have been focused on obesity late in life, which may have another impact on PC than obesity early in life. In the study of Robson et al. [28] for example, men having obesity between the ages of 20 and 29 years were less prone to develop PC $(\mathrm{OR}=0.3695 \% \mathrm{CI}=0.18-0.71)$ than nonobese men. The Health Professionals Follow-up, a prospective cohort study of 51,529 U.S. patients, also addressed the effect of obesity before age 30 and found an inverse relation as well $(\mathrm{RR}=0.5295 \% \mathrm{CI}=0.33-0.83)$ [29]. These analyses support the concept that the preadult hormonal milieu might be important to PC risk. However, an Australian populationbased, case-control study, with 1.476 cases and 1.409 controls, reported no association between BMI at age 21 and the risk of developing PC later in life [30]. These discrepancy can be caused due to different cutpoints used to define large body size and the use of different study populations (American vs. Australian).

Another difficulty comparing above-mentioned epidemiologic studies is that they usually consider PC as one biologic entity when it is very likely that PC is biologically heterogeneous, not only in terms of grade and stage but also in respect to its clinical behaviour [31].

In summary, although a link between obesity and the development of PC could be expected based on endocrine changes, the results of large studies were inconsistent due to methodological problems. Unfortunately they do not permit definitive conclusions and it demonstrates the difficulty to interpret the epidemiologic data when it comes to obesity and PC risk. However, there is a suggestion that obesity early in life decreases PC risk, where as obesity later in life increases the risk.

\section{The detection of prostate cancer in obese patients}

Prostate needle biopsy based on abnormal digital rectal examination (DRE) or elevated prostate specific antigen (PSA) level, is the cornerstone in the detection of PC. Obviously, factors that alter PSA and, or DRE can influence the detectability of PC, unrelated to cancer biology.

In obese patients it is more difficult to perform a proper DRE, so a palpable nodule can be missed. Nowadays, however, most PC are detected on biopsy following an abnormal PSA. As PSA production is androgen-dependent, it stands to reason that significant changes in androgens, which may result from obesity, could lower PSA without affecting the true incidence of PC. At the other hand, if obesity would result in larger prostates, this would make detection of a cancer at biopsy less likely, given an equalsize tumour.

Freedland et al. [32] tested the hypothesis, that obesity results in larger prostates and lower PSA levels, which delays the biopsy indication, in 1,414 men undergoing radical prostatectomy (RP). Obesity was significantly associated with increasing prostate weight, only in men younger than 63 years old, while there was no significant association between BMI and PSA. Assuming equal PSA concentrations the degree of prostatic enlargement in younger obese in this study would be expected to result in a modest (20$25 \%$ ) decrease for detecting PC due to technical issues relating to larger prostate size.

In a study of a referral-based biopsy population in the United States, Presti et al. [33] clearly focused a relation between BMI and PC detection on 787 patients. When stratified by age, significant higher detection rates were seen in the normal BMI group, compared to the obese group for men younger than 70 years (52 vs. $42 \%$ ), more likely because a normal BMI was associated with larger tumour volume on the biopsy irrespective of age. Conversely, in a referral-based biopsy population from Asia, consisting of 497 men, no significant association between BMI groups and PC detection was established [34]. Differences in racial distribution, population selection, duration of obesity, amount of biopsy cores and study size could very well explain this disparity.

Alternatively obesity may influence cancer screening behaviour, as shown for cervical and breast cancers [35]. Differences in PC screening behaviour may explain PC outcomes in obese men. Two recent American studies found 
that overweight and obese men are more likely to receive a PSA test than their counterparts in the healthy weight range [36, 37]. In these studies the odds for obese men having a PSA test was $1.26(95 \% \mathrm{CI}=1.06-1.36)$ respectively, 1.37 (95\% CI $=1.24-1.51$ ) higher than the odds for normal weight men.

In summary, although lower PSA levels and larger prostates seen in obese patients may decrease the detection rate of PC in obese men, on the other hand, they are more likely to receive a PSA test. So, overall impact of obesity on PC detection remains to be proved in clinical series.

\section{Influences of obesity on surgical outcome}

It is generally held that obesity makes many urological procedures more technically challenging. In RP this, for example, is the case for a higher likelihood of postoperative complications such as postoperative incontinence or stricture of the anastomosis. However, few data are available on the exact impact of obesity on the different surgical techniques. Of the available studies many consists of a small number of patients.

Only three reports investigated the impact of obesity and postoperative incontinence [38-40]. These retrospective studies reported no association between obesity and incontinence. Of note, all studies used questionnaires to evaluate the incontinence with a response rate of only 55 and $70 \%$, which may bias the results.

Three studies analysed the relation between obesity and health related quality of life (HRQOL) before and after RP. Two studies, with 422, respectively 672 men, concluded that excess BMI did not cause a decrease in HRQOL after RP [41, 42]. While a recent prospective study of 472 men found that high BMI did adversely affect HRQOL before and after the RP [43]. Although the populations of the three studies were approximately comparable with $20 \%$ obese patients, use of different questionnaires, regional variation in practitioner expertise and patient factors may affect these outcomes.

Despite surgical techniques blood loss remains a common intraoperative complication associated with RP, making a RP more difficult. If obese patients lose more blood during the operation, poorer vision may be one of the factors that obese patients are more prone to develop complications due to technical difficulty during the surgical dissection. However, in only one retrospective analysis of 436 men, obese men were likely to lose significant more blood during a RP, compared to non-obese men [44].

An alternative method of approach for RP in obese patients could be the perineal one. Three studies addressed the feasibility of radical perineal prostatectomy in obese patients. Small case series by Bockzo [45] and Dahm et al.
[46] consisted of 7 and 18 obese patients, respectively, have suggested that perineal RP is both safe and feasible, not only in obese, but also morbidly obese patients. The most recent controlled study of 71 severely obese and 71 nonobese patients, however, did find an increased risk of both surgical and anesthesia-related perioperartive complications, e.g. laryngospasm requiring reintubation, myocardial infarction, rectal injury and pulmonary edema [47]. Overall, a total of 12 complications (7 minor and 5 major) occurred in the obese group and 5 ( 2 minor and 3 major) occurred in the control group. This study did not provide information about late complications and functional outcomes.

The most recent surgical approach is laparoscopy. In a prospective study no significant differences were seen between operative and peroperative morbidity in obese patients who underwent a laparoscopic prostatectomy compared to nonobese men [48]. Although prostatectomy in obese patients was found more challenging due to the significant amount of intra-abdominal tissue, also the continence rate did not have any correlation with BMI or prostate size. Except for a longer mean operative time in obese patients, similar conclusions were reported by Brown et al. [49] and Eden et al. [50].

Another form of laparoscopic surgery is robotic assisted surgery. Two reports, showed the impact of obesity on robotic assisted laparoscopic prostatectomy. In a prospective study reported by Mikhail et al. [51] obesity did not increase perioperative and postoperative morbidity, except for operative time and estimated blood loss. After 1 year of followup, $74 \%$ of the obese patients returned to baseline continence. However, Ahlering et al. [52] reported significantly more complications in obese men and they required more time to return to baseline urinary function. At 6 months only $47 \%$ of obese patients had achieved urinary continence, which is low compared to the report of Mikhail et al. [51] Maybe the different length of followup can explain the discrepancy of the continence rate between both studies.

In summary, the surgical outcome of open RP indeed seems to be worse in obese patients. Since this appears less in laparoscopic RP, possible postoperative complications can affect the decision of choosing a therapy.

\section{Biochemical recurrence after surgery in obese patients}

Although the majority of men newly diagnosed with PC will have early-stage disease, little is known about the impact of obesity on oncological outcomes of primary therapy for clinically localized disease. Compared to non-obese men, two recent large American multi-institutional studies addressed a significant higher biochemical failure rate among obese men treated with RP. In the study of Amling 
et al. [53] obese patients had higher preoperative PSA levels and significantly higher-grade cancers than those not considered obese. Interestingly, several racial observations were made. In comparison with whites, blacks were significantly more likely to be obese, had significant higher PSA levels, and had significant higher-grade cancers. They were also more likely to have positive surgical margins at the time of RP. Overall, Univariate analysis, increasing BMI and black race were both associated with higher PSA and recurrence rates. In a multivariate analysis, however, only black race remained a significant independent predictor of cancer recurrence. In the study of Freedland et al. [54] obesity was also significantly related to race. BMI, whether categorical or continuous, was a significant predictor of biochemical recurrence and was associated with highergrade tumours. During the study period of 15 years there was a steady increase in mean BMI at time of surgery (from 26 to $28.5 \mathrm{~kg} / \mathrm{m}^{2}$ ), however in the PSA era, no significant relationship between year of surgery and PSA failure was seen. Although previous studies found a positive relation between obesity and biochemical recurrence, Mallah and co-workers [55], found a weak associations between disease progression and increasing BMI, which was of negligible prognostic value in men who received surgery. However, the definition of disease progression used, as well as the statistical approach, makes their results hard to compare with the previous mentioned reports. The results of these studies clearly indicate that even if obesity has a relation with biochemical progression, this is a multifactorial process.

There are some potential explanations for a higher biochemical recurrence rate in obese men. Oefelen et al. found intraoperative blood loss, which is likely to be more in obese patients [44], as an independent risk factor for PC recurrence [56]. This can be a causal relation, since perioperative transfusion with allogeneic blood may impair immune response and has been proved to have a detrimental effect on the recurrence of curable colorectal cancers [57]. This also may play a role in the higher biochemical failure rates seen in obese patients with PC. It is possible that the tamponade effect of the pneumoperitoneum and magnification of the scope during laparoscopy in obese patients may allow better hemostasis compared to the open surgical approaches. However, despite the possible clinical relevance, no comparing data are available.

Compared to non-obese, more positive surgical margins were seen in obese men who underwent a RP [53, 54]. This may be due to technical difficulty during the surgical dissection of the prostate among obese men. Higher rates of positive surgical margins could be responsible for the higher PSA recurrence rate seen in obese patients. However, in a study of 731 men who had an organ confined disease and negative surgical margins, the surgical technique was not the only factor to explain the worse outcomes among obese men, and the authors suggest that obesity was associated with a biologically more aggressive form of PC [58].

In summary, various studies suggested that obesity may result in a higher recurrence rates after RP. The question remains, whether the differences in outcome are predominantly driven by worse biological features or technical difficulties during the surgical procedure.

\section{Conclusion}

Obesity is a growing problem in Western countries. Autopsy studies from numerous countries worldwide, however, have shown similar rates of latent or clinically insignificant PC, despite markedly different clinical significant PC and PC death rates among these populations [59, 60]. These findings suggest that although clinically insignificant PC may be common to all races and ethnic groups, some as yet unknown factor or factors may promote progression into clinically significant cancers. Recent international trends show that PC incidence is increasing in low-incidence countries such as China and Japan. At the same time these countries are adopting westernised lifestyles associated with higher rates of obesity. These observations suggest that there is a relation between obesity and PC. In any case, epidemiological studies are showing conflicting results between obesity and developing prostate diseases. In contrast, more consistent findings have linked obesity with PC mortality, advanced stage and higher gleason score.

Although any relation between obesity and $\mathrm{PC}$ remains to be proven, more prospective studies are needed to examine the most optimal treatment modality, which means the best oncological and functional results, for obese patients suffering from PC.

In all, the relationship between obesity and prostate cancer is currently a hotly debated topic, but despite the number of publications devoted to the topic, the actual nature of the relationship remains uncertain, and one should bear in mind that obesity is a modifiable lifestyle factor.

\section{References}

1. James PT, Rigby N, Leach R (2004) International obesity task force. The obesity epidemic, metabolic syndrome and future prevention strategies. Eur J Cardiovasc Prev Rehabil 11(1):3-8

2. Calle EE, Kaaks R (2004) Overweight, obesity and cancer: epidemiological evidence and proposed mechanisms. Nat Rev Cancer 4:579-591

3. Håheim LL, Wisløff TF, Holme I, Nafstad P (2006) Metabolic syndrome predicts prostate cancer in a cohort of middle-aged Norwegian men followed for 27 years. Am J Epidemiol 164:769-774

4. Mokdad AH, Ford ES, Bowman BA et al (2003) Prevalence of obesity, diabetes, and obesity-related health risk factors, 2001. JAMA 289:76-79 
5. Calle EE, Rodriguez C, Walker-Thurmond K, Thun MJ (2003) Overweight, obesity, and mortality from cancer in a prospectively studied cohort of U.S. adults. N Engl J Med 348:1625-1638

6. Gapstur SM, Gann PH, Kopp P et al (2002) Serum androgen concentrations in young men: a longitudinal analysis of associations with age, obesity, and race. The CARDIA Male Hormone Study. Cancer Epidemiol Biomarkers Prev 11:1041-1047

7. Isom-Batz G, Bianco Jr FJ, Kattan MW, Mulhall JP, Lilja H, Eastham JA (2005) Testosterone as a predictor of pathological stage in clinically localized prostate cancer. J Urol 173:1935-1937

8. Imamoto T, Suzuki H, Fukasawa S et al (2005) Pretreatment serum testosterone level as a predictive factor of pathological stage in localized prostate cancer patients treated with radical prostatectomy. Eur Urol 47:308-312

9. Stattin P, Lumme S, Tenkanen L et al (2004) High levels of circulating testosterone are not associated with increased prostate cancer risk: a pooled prospective study. Int J Cancer 108:418-424

10. Hsing AW, Reichardt JK, Stanczyk FZ (2002) Hormones and prostate cancer: current perspectives and future directions. Prostate 52:213-235

11. Vona-Davis L, Vedula G, McFadden DW, VanGilder R, Somasundar SMP, Hileman SM (2003) Identification of leptin receptor isoforms in human prostate cancer cells. J Surg Res 114:302

12. Somasundar P, Yu AK, Vona-Davis L, McFadden DW (2003) Differential effects of leptin on cancer in vitro. J Surg Res 113:5055

13. Onuma M, Bub JD, Rummel TL, Iwamoto Y (2003) Prostate cancer cell-adipocyte interaction. Leptin mediates androgen independent prostate cancer cell proliferation through c-jun NH2-terminal kinase. J Biol Chem 278:42660-42667

14. Hsing AW, Chua Jr S, Gao YT et al (2001) Prostate cancer risk and serum levels of insulin and leptin: a population-based study. $\mathbf{J}$ Natl Cancer Inst 93:783-789

15. Stattin P, Kaaks R, Johansson R, Gislefoss R et al (2003) Plasma leptin is not associated with prostate cancer risk. Cancer Epidemiol Biomarker Prev 12:474-475

16. Saglam K, Aydur E, Yilmaz M, Goktas S (2003) Leptin influences cellular differentiation and progression in prostate cancer. J Urol 169:1308-1311

17. Chang S, Hursting SD, Contois JH et al (2001) Leptin and prostate cancer. Prostate 46:62-67

18. Freedland SJ, Sokoll LJ, Mangold LA et al (2005) Serum leptin and pathological findings at the time of radical prostatectomy. $\mathrm{J}$ Urol 173:773-776

19. Pollak M, Beamer W, Zhang JC (1999) Insulin-like growth factors and prostate cancer. Cancer Metastasis Rev 17:383-390

20. Stattin P, Rinaldi S, Biessy C, Stenman UH, Hallmans G, Kaaks R (2004) High levels of circulating insulin-like growth factor-1 increase prostate cancer risk: a prospective study in a populationbased nonscreened cohort. J Clin Oncol 22:3104-3112

21. Chan JM, Stampfer MJ, Giovannucci E et al (1998) Plasma insulin-like growth factor-1 and prostate cancer risk: a prospective study. Science 279:563-566

22. Wolk A, Mantzoros CS, Andersson SO et al (1998) Insulin-like growth factor 1 and prostate cancer risk: a population-based, casecontrol study. J Natl Cancer Inst 90:911-915

23. Andersson SO, Wolk A, Bergstrom R et al (1997) Body size and prostate cancer: a 20-year follow-up study among 135006 Swedish construction workers. J Natl Cancer Inst 89(5):385-389

24. Engeland A, Tretli S, Bjørge T (2003) Height, body mass index, and prostate cancer: a follow-up of 950000 Norwegian men. Br J Cancer 89:1237-1242

25. Bradbury BD, Wilk JB, Kaye JA (2005) Obesity and the risk of prostate cancer (United States). Cancer Causes Control 16:637641
26. Porter MP, Stanford JL (2005) Obesity and the risk of prostate cancer. Prostate 62:316-321

27. Jonsson F, Wolk A, Pedersen NL et al (2003) Obesity and hormone-dependent tumors: cohort and co-twin control studies based on the Swedish twin registry. Int J Cancer 106:594-599

28. Robinson WR, Stevens J, Gammon MD, John EM (2005) Obesity before age 30 years and risk of advanced prostate cancer. Am J Epididemiol 161:1107-1114

29. Giovannucci E, Rimm EB, Liu Y et al (2003) Body mass index and risk of prostate cancer in U.S. health professionals. J Natl Cancer Inst 95:1240-1244

30. Giles GG, Severi G, English DR et al (2003) Early growth, adult body size and prostate cancer risk. Int J Cancer 103:241-245

31. Miller GJ, Torkko KC (2001) Natural history of prostate cancerepidemiologic considerations. Epidemiol Rev 23:14-18

32. Freedland SJ, Platz EA, Presti JC et al (2006) Obesity, serum prostate specific antigen and prostate size: implications for prostate cancer detection. J Urol 175:500-504

33. Presti JC Jr, Lee U, Brooks JD, Terris MK (2004) Lower body mass index is associated with a higher prostate cancer detection rate and less favorable pathologic features in a biopsy population. J Urol 171:2199-2202

34. Kobayashi T, Mitsumori K, Nishizawa K, Kawahara T, Ogura K, Ide Y (2005) Association between body mass index and prostate cancer detection rates in Japanese urologic patients. Urology 66:130-134

35. Wee CC, McCarthy EP, Davis RB, Phillips RS (2000) Screening for cervical and breast cancer: is obesity an unrecognized barrier to preventive care?. Ann Intern Med 132:697-704

36. Fontaine KR, Heo M, Allison DB (2005) Obesity and prostate cancer screening in the USA. Pub Health 119:694-698

37. Scales CD Jr, Curtis LH, Norris RD, Schulman KA, Dahm P, Moul JW (2007) Relationship between body mass index and prostate cancer screening in the united states. J Urol 177:493-498

38. Hsu EL, Hong EK, Lepor H (2003) Influence of body weight and prostate volume on intraoperative, perioperative, and postoperative outcomes after radical retropubic prostatectomy. Urology 61:601-606

39. Mulholland TL, Huynh PN, Huang RR, Wong C, Diokno AC, Peters KM (2006) Urinary incontinence after radical retropubic prostatectomy is not related to patients body mass index. Prostate Cancer Prostatic Dis 9(2):1-7

40. Wille S, Heidenreich A, von Knobloch R, Hofmann R, Engelmann $\mathrm{U}$ (2006) Impact of comorbidities on post-prostatectomy incontinence. Urol int 76:223-226

41. Anast JW, Sadetsky N, Pasta DJ et al (2005) The impact of obesity on health related quality of life before and after radical prostatectomy (data from CaPSURE). J Urol 173:1132-1138

42. Freedland SJ, Haffner MC, Landis PK, Saigal CS, Carter HB (2005) Obesity does not adversely affect health-related quality-oflife outcomes after anatomic retropubic radical prostatectomy. Urology 65:1131-1136

43. Montgomery JS, Gayed BA, Hollenbeck BK, Daignault S, Sanda MG, Montie JE, Wei JT (2006) Obesity adversely affects health related quality of life before and after radical retropubic prostatectomy. J Urol 176:257-262

44. Chang SS, Duong DT, Wells N, Cole EE, Smith Jr JA, Cookson MS (2004) Predicting blood loss and transfusion requirements during radical prostatectomy: the significant negative impact of increasing body mass index. J Urol 171:1861-1865

45. Boczko J, Melman A (2003) Radical perineal prostatectomy in obese patients. Urology 62:467-469

46. Dahm P, Yang BK, Salmen CR, Moul JW, Gan TJ (2005) Radical perineal prostatectomy for the treatment of localized prostate cancer in morbidly obese patients. J Urol 174:131-134 
47. Yang BK, Gan TJ, Salmen CR, Cancel QV, Vieweg J, Dahm P (2006) Radical perineal prostatectomy for treatment of localized prostate cancer in obese and nonobese patients: a matched control study. Urology 67:990-995

48. Singh A, Fagin R, Shah G, Shekarriz B (2005) Impact of prostate size and body mass index on perioperative morbidity after laparoscopic radical prostatectomy. J Urol 173:552-554

49. Brown JA, Rodin DM, Lee B, Dahl DM (2005) Laparoscopic radical prostatectomy and body mass index: an assessment of 151 sequential cases. J Urol 173:442-445

50. Eden CG, Chang CM, Gianduzzo T, Moon DA (2006) The impact of obesity on laparoscopic radical prostatectomy. BJU Int 98:1279-1282

51. Mikhail AA, Stockton BR, Orvieto MA et al (2006) Robotic-assisted laparoscopic prostatectomy in overweight and obese patients. Urology 67:774-779

52. Ahlering TE, Eichel L, Edwards R, Skarecky DW (2005) Impact of obesity on clinical outcomes in robotic prostatectomy. Urology 65:740-744

53. Amling CL, Riffenburgh RH, Sun L et al (2004) Pathologic variables and recurrence rates as related to obesity and race in men with prostate cancer undergoing radical prostatectomy. J Clin Oncol 22:439-45
54. Freedland SJ, Aronson WJ, Kane CJ et al (2004) Impact of obesity on biochemical control after radical prostatectomy for clinically localized prostate cancer: a report by the shared equal access regional cancer hospital database study group. J Clin Oncol 22:446453

55. Mallah KH, DiBlasio CJ, Rhee AC, Scardino PT, Kattan MW (2005) Body mass index is weakly associated with, and not a helpful predictor of, disease progression in men with clinically localized prostate carcinoma treated with radical prostatectomy. Cancer 103:2030-2034

56. Oefelein MG, Colangelo LA, Rademaker AW, McVary KT (1995) Intraoperative blood loss and prognosis in prostate cancer patients undergoing radical retropubic prostatectomy. J Urol 154:442-447

57. Amanto A, Pescatori M (2006) Perioperative blood transfusions for the recurrence of colorectal cancer (review). The Cochrane Database of Systematic Reviews Issue 1, Art. No.:CD005033.pub2

58. Freedland SJ, Terris MK, Presti Jr JC et al (2004) Obesity and biochemical outcome following radical prostatectomy for organ confined disease with negative surgical margins. J Urol 172:520-524

59. Breslow N, Chan CW, Dhom D et al (1977) Latent carcinoma of the prostate in seven areas. Int J Cancer 20:680-688

60. Yatani R, Chigusa I, Akazaki K et al (1982) Geographic pathology of latent prostatic carcinoma. Int J Cancer 29:611-616 